\title{
Attribute-Driven Granular Model for EMG-based Pinch and Fingertip Force Grand Recognition
}

\author{
Yinfeng Fang, Member, IEEE, Dalin Zhou, Student Member, IEEE, Kairu Li, Student Member, IEEE, Zhaojie Ju, \\ Senior Member, IEEE, Honghai Liu*, Senior Member, IEEE
}

\begin{abstract}
Fine multi-functional prosthetic hand manipulation requires precise control on the pinch-type and the corresponding force, and it is a challenge to decode both aspects from myoelectric signals. This study proposed an attribute-driven granular model (AGrM) under a machine learning scheme to solve this problem. The model utilises the additionally captured attribute as the latent variable for a supervised granulation procedure. It was fulfilled for EMG-based pinch-type classification and the fingertip force grand prediction. In the experiments, sixteen channels of surface electromyographic signals (i.e. main-attribute) and continuous fingertip force (i.e. sub-attribute) were simultaneously collected while subjects performing eight types of hand pinches. The use of AGrM improved the pinch-type recognition accuracy to around $97.2 \%$ by $1.8 \%$ when constructing eight granules for each grasping type, and received more than $90 \%$ force grand prediction accuracy at any granular level greater than six. Further sensitivity analysis verified its robustness with respect to different channel combination and interferences. In the comparison with other clustering-based granulation methods, AGrM achieved comparable pinch recognition accuracy but was of lowest computational cost and highest force grand prediction accuracy.
\end{abstract}

\section{INTRODUCTION}

sEMG based pinch recognition can be utilised to facilitate prosthetic hand manipulation via the use of machine learning technology [1]-[4]. To promote the flexibility of object manipulation, advanced multi-functional robotic hands with force sensors on finger tips have been developed, which arouses the need for the control of both the gesture and the force. However, constrained by the performance of intuitive humanmachine interface, it is sill difficult to be implemented from the perspective of amputees. The change of muscle contraction force is usually considered as a negative factor for EMG-based hand motion recognition. However, dexterous multifunctional prosthetic hand control requires the synergy of robust hand motion prediction and accurate force estimation [5], [6]. Fig. 1 illustrates that both gesture and force control are required

This work was supported by the 7th framework programme of the European Union under Grant 600915 (Development of Robot-enhanced Therapy for Children with Autism Spectrum Disorders, DREAM) and National Natural Science Foundation of China under Grants 51575338, 51575407 and 51475427

Fang, Y. is with the School of Communication Engineering, Hanzhou Dianzi University, Hangzhou, 310018, China.

Zhou, D., Li, K., Ju, Z. and Liu, H., are with the Intelligent Systems and Biomedical Robotics Group, School of Computing, University of Portsmouth, Portsmouth, PO1 3HE, U.K.

Liu, H. is also with the State Key Laboratory of Mechanical System and Vibration, School of Mechanical Engineering, Shanghai Jiao Tong University, Shanghai, 200240, China.

The corresponding author is Liu, H. (honghai.liu@icloud.com)

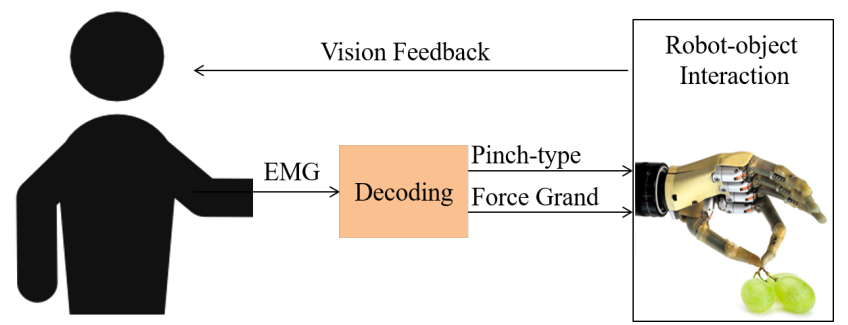

Fig. 1: The scenario of pinch-type and fingertip force grand prediction for myoelectric control.

in a human machine interaction scenario. Conventionally, a classifier is not able to implement EMG-based hand gesture identification as well as force estimation. The current study aims to fill the gap by means of AGrM, fusing pinch recognition and force estimation in a unified machine learning framework.

The EMG pattern of the same grasp under different grasping force mismatches with each other, and it leads to the misclassification of pattern recognition systems [3], [7]-[9]. The presence of contractions from unseen force levels increased the error considerably by more than $32 \%$, and to counteract the severe degradation, a pooled training set comprising all force levels was suggested [3]. Let alone the utilization of a pooled training set, new feature extraction strategy immunising to dynamic muscle contraction were investigated [7], [10]. Fang, et al [11] proposed a granular model by the use of force information to improve the motion recognition accuracy. Alternatively, Powell, et al. [8] and Fang, et al. [12] resorted to user-training to unify users' muscle contraction to improve hand motion recognition accuracy. These studies have achieved the great success in improving the robustness of hand gesture recognition, but they ignore the other equally important aspect: force estimation.

A precise force control resides the base of object manipulation for both intact people and prosthetic hand users. It has been highlighted that portable force control could increase amputees' acceptance rate for myoelectric forearm prostheses [4]. Recently, Wu et al. employed a generalised regression neural network to estimate grip force for the enhancement of dexterous control of prosthetic hand [13]. The advancement of sensitive tactile sensors and sophisticated grasp strategies could contribute to relatively stable functionality, but natural human-machine interaction requires an anthropopathic prosthetic hand. Thus, intuitively, more endeavors should be drawn on tracing the force directly from the physiological 
information of the users, which can enhance the extended physiological proprioception and increase the feeling of bodyownership [14].

In the literature, EMG-based hand grasp/pinch and its force prediction are mostly studied as two separate topics. Castellini et al. [15] presented a study on the recognition of 5 types of grasps together with force estimation using artificial neural networks (ANN), support vector machine (SVM) and locally weighted projection regression (LWPR), achieving grasp recognition accuracy of $89.67 \pm 1.53 \%$ and force prediction error of $7.89 \pm 0.09 \%$. Yang et al. [14], [16] utilised SVM for hand gesture recognition (27 classes) and $\varepsilon$ support vector regression ( $\varepsilon-S V R$ ) to estimate the grasping force under three hand grasps instead of the whole gesture candidate set. These studies still employed pattern recognition approaches for grasping-type recognition and regression models for force estimation in a sequential order. A major problem of these strategies is that sequential computing would result in manipulation delay for prosthetic hand manipulation. It is well known that latency is critical in improving the acceptance rate of amputees. The current study aims to unify EMG-driven pinch recognition and force estimation in one machine learning framework to avoid the potential delay and to simplify the algorithm training procedure.

The primary contribution is to resolve the bottleneck problem in simultaneously predicting the hand gestures and the applied force from the sEMG signal for prosthetic hand manipulation via a GrM variant (i.g. AGrM). Secondly the GrM variant is developed to predict the hand pinch type as well as the fingertip force applied to an object, allowing the use of additional attribute (i.e. force) to enhance the performance of gesture recognition accuracy via a supervised granulation approach. Our experiments demonstrated its success in enhancing the pinch type recognition accuracy as well as force grand prediction accuracy under a variety of interference.

The remaining of this paper is organised as follows. Section II summarised the state-of-the-art of granular computing $(\mathrm{GrC})$ in machine learning and theoretically introduced AGrM in details. Section III illustrated experimental setup for data collection, and introduced the methods for sEMG feature extraction and procedure for performance evaluation. Section IV disclosed the experimental results with discussions. Section $\mathrm{V}$ concluded the paper with future work.

\section{Attribute-Driven GRAnUlar Model For PATtern RECOGNITION}

\section{A. Granular Computing in Machine Learning}

Granular computing ( $\mathrm{GrC}$ ) solves problems via making use of granules, i.e. groups, classes or clusters of a universe, which is closely related to the cognitive strategy of human being in problem solving and it is technically transferable to the design of human-centric intelligent systems [17], which has been applied in image-based crowd segmentation [18], longterm prediction model for the energy system [19], [20], video based object tracking [21] and principle curve extraction [22], etc.

Inspired from the concept of $\mathrm{GrC}$, several novel classifiers were designed. Liu, et al. [23] proposed a granular computing classification algorithm based on distance measurement, where granules were constructed according to geometric shapes. Roh, et al. [24] proposed a methodology for designing granular fuzzy classifiers based on information granularity. Both methods split the entire input space into a collection of subspaces to develop rule-based classifiers. However, without sufficient comparison and theoretical proof, their performances are still in doubt.

Besides, GrC was also employed to extend conventional classifiers. Yuchun, et al. [25] built a granular SVM classifier by building a sequence of information granules. Instead of explicitly using the concept of information granulation of $\mathrm{GrC}$, Zhu, et al. [26] proposed a study on subclass discrimination analysis, where granule construction and LDA classification were merged theoretically. Both methods leverage information granulation to solve nonlinear and inseparable problem by strategies aiming at linearly separable subproblems.

As a significant part of $\mathrm{GrC}$, information granulation is critical to formulate the rules for granule construction, which is also being known as granular mapping in [27]. A general criterion for granule construction is to draw elements with indistinguishability, similarity, proximity or functionality together [28]. Traditional granulation methods adopt unsupervised clustering algorithms, such as Nearest Neighbour (NN) [26], K-Means [29], [30], hierarchical clustering [31], spatial partition trees [32], fuzzy C-means [20], [22], [33], [34], and prototype-based optimization [35], to construct granules. The unsupervised algorithm ensures the elements with certain similarity to be assembled into one granule, however the granules are non-interpretable in the view of semantic context.

From a novel perspective, our study proposes a supervised granulation method (i.g. attribute driven granulation) in which granules were generated according to the status of additional attributes rather than the use of unsupervised clustering approach. Consequently, each granule is interpretable by the attributes. It provides a practical solution that how to utilize additional information to enhance a training procedure, in despite of that the information may not be provided during prediction. Back to the topic of EMG-driven motion recognition, it is practical to obtain additional sensory information via placing sensors on hands or objects to collect a training dataset [36], [37]. However, the demand of real application may only allows the use of EMG signal. Thus, this study raises a common problem in machine learning in terms of how to utilise additional sensory signals to enhance performance of a machine learning system.

\section{B. Training Dataset Granulation}

Given a training dataset $\mathcal{D}$ of $K$ training samples from $l$ distinct classes: $\mathcal{D}=\left\{D_{i}, i=1, \ldots, l\right\}=\left\{\left(\mathbf{x}_{k}, y_{k}\right), k=\right.$ $1 \ldots K\}$, where $\mathbf{x}_{k} \in \boldsymbol{R}^{n}$ and $y_{k}$ labels $\mathbf{x}_{k}$ to one of classes in $\Omega_{c}=\left\{c_{1}, c_{2}, \ldots, c_{l}\right\}$. A classifier is trained by the dataset to fit a mapping rule: $f: \mathbf{x} \rightarrow c$ that assigns a class label $c \in \Omega_{c}$ to an input described by a set of attributes $\mathbf{x}=\left\{x_{1}, x_{2}, . . x_{n}\right\} \in \mathbf{R}^{n}$.

According to the theory of information granulation, one class can be decomposed into subclass granules. In spite 


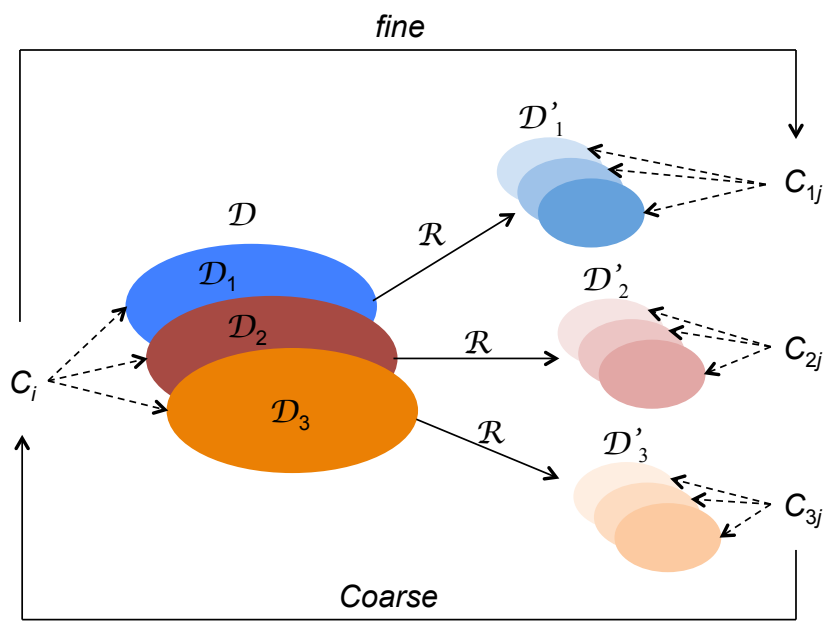

Fig. 2: Transfer an original 3-class dataset to a granulised dataset via information granulation rule $\mathcal{R}$.

of any granulation rule $\mathcal{R}$, a granular model divides the original data set to a new one with extended classes, also called subclass granules. The new dataset can be denoted by $\mathcal{D}^{\prime}=\left\{D_{i}^{\prime}, i=1, \ldots, l\right\}=\left\{\left(\mathbf{x}_{k}, y_{k}^{\prime}\right), k=1, \ldots, K\right\}$, where $y_{k}^{\prime}$ labels $\mathbf{x}_{k}$ to one of $l \times m$ classes in $\Omega_{c}^{\prime}=\left\{\Omega_{c 1}, \Omega_{c 2}, \ldots, \Omega_{c l}\right\}$ and $\Omega_{c i}=\left\{c_{i 1}, c_{i 2}, \ldots, c_{i m}\right\}(i=1,2, \ldots, l)$ is a set of granules decomposed from the entirety $c_{i}$. The relationship between $c_{i j}$ and $c_{i}$ can be denoted by $c_{i j} \leq c_{i}$, saying $c_{i j}$ is a granule of $c_{i}$, and this procedure can be obtained by a fining function,

$$
c_{i j}=\operatorname{fine}\left(c_{i}\right) .
$$

Accordingly, a classifier $f^{\prime}: \mathbf{x} \rightarrow c^{\prime}$ can be trained to classify any $x$ to one class in $\Omega_{c}^{\prime}$.

As described above, the granular model separates the training dataset that belong to one class into several sub-classes, and assigns each individual to granulised classes. Thus, a classifier can be trained with the granulised training dataset to solve a $\mathrm{lm}$-class classification problem. It can be recovered to a $l$-class problem through an organisation procedure by defining a coarsing function as

$$
c_{i}=\operatorname{coarse}\left(c_{i j}\right),
$$

indicating that any sample that belongs to class $c_{i j}$ also belongs to class $c_{i}$.

Fig. 2 illustrates the granulation procedure of a 3-class training dataset into $3 j$-class dataset by rule $\mathcal{R}$.

\section{Attribute-driven Granulation}

Training dataset granulation describes the fact that the observations in one class can be granulised into several subclass granules. Traditionally, clustering-based methods, like NN, KMeans algorithm and Gaussian mixture model (GMM), are used to assemble homogenised samples into a granule based on their similarity and geometry distance. However, granules generated by these unsupervised approaches are semantic inexplicable [38]. A supervised approach is proposed in the current study to implement information granulation. In the context of pattern recognition, this study considers the attributes in

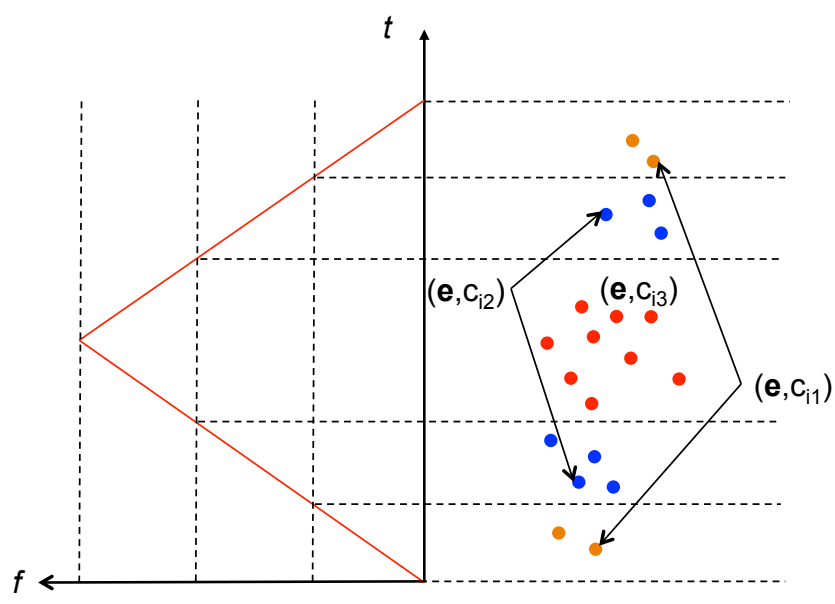

Fig. 3: The use of AGrM to separate EMG feature sets supervised by force information as the sub-attribute.

a dataset into two types: main-attributes and sub-attributes. Main-attributes exist in both training and testing dataset, while sub-attributes are only contained in a training dataset.

Considering the scenario of hand motion recognition, during training dataset collection, both EMG signal and fingertip force can be gathered via placing EMG sensors on the skin and force sensors on finger tips or objects. But during reallife application, amputees can only rely on EMG signal for prothetic hand manipulation. In this scenario, EMG signal is the main-attribute and force signal is the sub-attribute. Consequently, it brings us two concerns: 1) how can we utilise the sub-attribute to train a classifier that would better predict amputee's intention; 2) how to train a classier that can also predict the sub-attribute to some degrees. The proposed AGrM solves these two concerns as described below.

Given a training dataset $\mathcal{D}$, each observation o consists of three parts, sub-attribute $\mathbf{s}$, main-attribute $\mathbf{m}$ and class label $c$,

$$
\mathbf{o}=(\mathbf{s}, \mathbf{m}, c), \mathbf{o} \in \Omega .
$$

where $\Omega$ is the observation space.

A granulation operator is defined as the following equation,

$$
\mathcal{R}: \Omega \rightarrow \Omega^{\prime} ;(\mathbf{s}, c) \mapsto c^{\prime}
$$

where $\mathcal{R}$ is the rule for granulation, $\Omega^{\prime}$ is the space consisting of the divided subclass labels and $c^{\prime}$ is the class label after granulation.

In the case of EMG based pinch recognition (8 classes) and force grand prediction, the model can be embodied as

$$
\begin{gathered}
\mathbf{o}=(f, \mathbf{e}, c), \\
\mathcal{R}:(f, \mathbf{e}, c) \mapsto\left(\mathbf{e}, c^{\prime}\right)
\end{gathered}
$$

where $f$ is a one-dimensional contact force value between the hand and an object, $\mathbf{e}$ is the EMG feature vector; $c \in\left\{c_{i}\right\}$ and $c^{\prime} \in\left\{c_{i j}\right\}$, where $i=1,2, \ldots, 8$ and $j=1,2, \ldots, m . m$ is the total number of granules for each grasp, also indicating the force level of grasp. It is worth noting that this study predicts the force grand instead of the continuous force value, and the 
force grand is constrained by the predefined interval among the maximum and minimum force based on the training database.

To verify the AGrM, a crisp rule is tested in this study to construct $m$ granules for each class via defining the rule as follows

$$
\mathcal{R}\left\{\left(f, \mathbf{e}, c_{i}\right)\right\}=\left(\mathbf{e}, c_{i j}\right), f \in((j-1) s, j s]+f_{(i, \min )},
$$

where $\left.s=\frac{f_{(i, \max )}-f_{(i, \min )}}{m}, f_{(i, \min }\right)$ and $f_{(i, \max )}$ are the minimum and maximum force recorded in the training dataset for pinch type $c_{i}$. $\left(\mathbf{e}, c_{i j}\right)$ indicates that a EMG feature vector $\mathbf{e}$ corresponds to the $j^{\text {th }}$ force grand of the $i^{t h}$ pinch-type $c_{i}$. Fig. 3 sketches the granulation approach that divides the training dataset $\mathcal{D}_{i}$ into three granules $\left(\mathbf{e}, c_{i 1}\right),\left(\mathbf{e}, c_{i 2}\right),\left(\mathbf{e}, c_{i 3}\right)$.

\section{Evaluation Indicators}

To evaluate the efficiency of AGrM in the application of EMG based pinch-type and force grand prediction, this study defined several indicators for comparison purposes.

Two types of accuracy metrics are defined in this research: pinch recognition accuracy $\left(a c c_{g}\right)$ and force estimation accuracy $\left(a c c_{f}\right)$. Pinch recognition accuracy is defined as

$$
a c c_{g}=\frac{N_{g}}{N},
$$

where $N$ is the number of all samples for test, and $N_{g}$ is the correctly classified pinch, considering the label of $c_{i j}$ is correctly classified into $c_{\hat{i} \hat{j}}$, when $i=\hat{i}$, and $j$ can be different with $\hat{j}$.

Force grand prediction accuracy $\left(a c c_{f}\right)$ is defined as

$$
a c c_{f}=1-\frac{\sum w_{f}\left(c_{i j}, c_{\hat{i} \hat{j}}\right)}{N},
$$

where

$$
w_{f}\left(c_{i j}, c_{\hat{i} \hat{j}}\right)=\left\{\begin{array}{lll}
0, & \text { for } & i \neq \hat{i} \\
\frac{|j-\hat{j}|}{\alpha_{m}(m-1)}, & \text { for } & i=\hat{i}
\end{array},\right.
$$

in which $c_{i j}$ indicates the actual pinch-type and force grand, $c_{\hat{i} \hat{j}}$ is the predicted result and $m$ is the number of divided force levels. $\frac{|j-\hat{j}|}{\alpha_{m}(m-1)}$ is the punishment factor, in which

$$
\alpha_{m}=\frac{4}{m^{2}} \sum_{k=1}^{m-1} \frac{k}{m-1}(m-k), \quad m \geqslant 2 .
$$

A matrix consists of all punishment factors is called punishment matrix. Coefficient $\alpha_{m}$ is defined to ensure that the mean of punishment matrix is equal to 0.5 for different number of $m$. It ensures that the expectation of evenly distributed inputs $c_{\hat{i} \hat{j}}$ is equal for any value of $m$. The punishment matrix at $m=2,3$, is demonstrated in Fig. 4. Note that $a c c_{f}$ only calculates the estimation accuracy when the grasping-type is classified correctly.

Three types of computation cost is defined in this study, which are granulation time $\left(T_{\text {granulation }}\right)$, classifier training time $\left(T_{\text {training }}\right)$ and prediction time $\left(T_{\text {prediction }}\right)$. It is expected that the time complexity remains a stable level with the increase of the number of granules $(m)$. The computation cost on prediction and feature extraction $\left(T_{\text {feature }}\right)$ is related

\begin{tabular}{|c|c|c|}
\hline$\hat{j}=1$ & 0 & 1 \\
\hline$\hat{j}=2$ & 1 & 0 \\
\hline & $j=1$ & $j=2$ \\
\hline
\end{tabular}

(a)

\begin{tabular}{|c|c|c|c|}
\hline$\hat{j}=1$ & 0 & $9 / 16$ & $9 / 8$ \\
\hline$\hat{j}=2$ & $9 / 16$ & 0 & $9 / 16$ \\
\hline$\hat{j}=3$ & $9 / 8$ & $9 / 16$ & 0 \\
\hline & $j=1$ & $j=2$ & $j=3$ \\
\hline
\end{tabular}

(b)
Fig. 4: (a) punishment matrix at $m=2$; (b) punishment matrix at $m=3$.

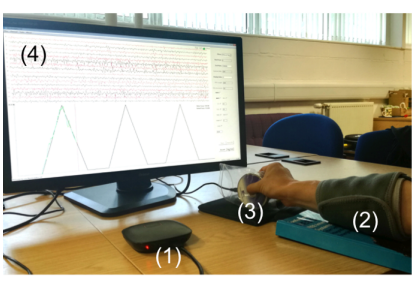

(a)

(c)

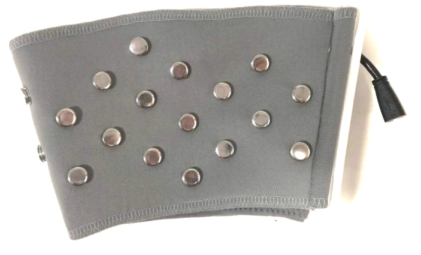

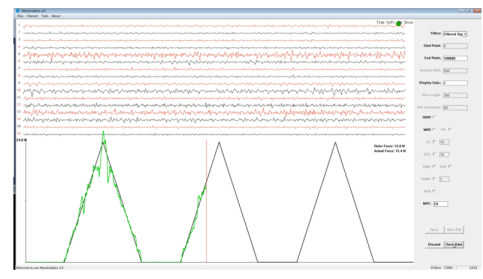

(b)
Fig. 5: Devices and experimental scenario.

to the real-time performance of any pattern recognition system. For a prosthetic hand control system, $T_{\text {prediction }}+T_{\text {feature }}$ should be less than the increment of the sliding window.

\section{Data Collection And Processing}

\section{A. Surface Electromyography}

An electrode armband was deigned to attach 18 sEMG electrode on the forearm forming 16 monopolar sEMG channels, as seen in Fig. 5 (a-2 and c). These electrodes were evenly distributed on a piece of cloth, and fabric sleeve, which ensures high quality of skin-electrode contact and also make it suitable for different forearm size, as seen in Fig. 5(c). An EMG device was also customised for the acquisition of EMG signal. The prototype can be seen in Fig. 5(a-1). The lowest resolution for the captured EMG signal is $5.72 \mu \mathrm{V}$ with 12 bits sampling resolution. The signal is filtered by a $20-500 \mathrm{~Hz}$ band-pass filter, and meanwhile a notch filter was also included to remove $50 \mathrm{~Hz}$ power-line interference. The sampling frequency was set to $1 \mathrm{kHz}$ in this study, which is higher than twice the commonly accepted maximum EMG signal frequency $400 \mathrm{~Hz}$ [39]. 


\section{B. Force Sensor}

The force sensor (UD-050-015-S*C01, Loadstar, US) was used as the object, and meanwhile it measured external pinch force applied to the sensor. Its capacity was $50 \mathrm{lb}$ at the resolution of $0.125 \mathrm{lb}$. The sampling frequency was adjusted to $25 \mathrm{~Hz}$. A software was customised to synchronously capture both types of sensory signal and to display the force hint as required in the experiment.

\section{Data Collection}

Eight able-bodied subjects volunteered for the experiment. The entire experimental procedure was introduced to them in oral and written form, following which their consent was taken in writing. The experiment was approved by the Ethical Committee of University of Portsmouth. The subjects were seated comfortably on an adjustable office chair for the entire duration.

In an initial round of data collection, we measured the maximal forces that each subject could apply for each pinch. These pinches included Tip Pinch (G1), three Tip Pinch Variants (G2, G3, G4), opposing the middle, ring,and little finger to the thumb, respectively, Extension Type (G5), Parallel Extension(G6), Tripod (G7), and Lateral (G8), as can be seen in Fig. 5(d). The selection of these motions followed the Feix's taxonomy [40], and meanwhile considered the executability by the dexterous self-powered hand prostheses, named i-LIMB Ultra Revolution. For each pinch, the subjects were asked to follow a predefined force hint that linearly increases to $60 \%$ maximal voluntary contraction (MVC) in ten seconds and decreases to $0 \% \mathrm{MVC}$ in the following ten seconds, repeated three times. Fig. 5 (a) demonstrated the experimental scenario, in which (1) is the wireless sEMG acquisition device, (2) is the electrode armband, (3) is the force sensor and (4) is the graphic user interface for signal display and recording. Fig. 5 (b) demonstrated that both sEMG and force signals, as the subjects were following the force hint to apply proper pinch force on the sensor.

\section{EMG Feature Extraction and Segmentation}

In each pinch, the EMG signals were segmented into a series of $200 \mathrm{~ms}$ windows and $40 \mathrm{~ms}$ overlap, which ensured that the extracted feature was of the same frequency as the force signal. It also required that the time consumption of feature extraction and motion prediction should be restricted within $40 \mathrm{~ms}$ to guarantee a real-time performance. Hudgins' time domain sEMG feature set [12], [41], [42], including mean absolute value (MAV), waveform length (WL), zero changes (ZC), slope sign changes (SSC), was selected in this study; hence, the dimensionality of a feature vector is 64 for 16 EMG channels. Furthermore, the samples within ranges of [10s, 30s], [40s, 60s] and [70s, 90s] with pinch motion were extracted as the dataset for further analysis. As a result, the dataset from a subject would include 11976 samples with 8 pinches.

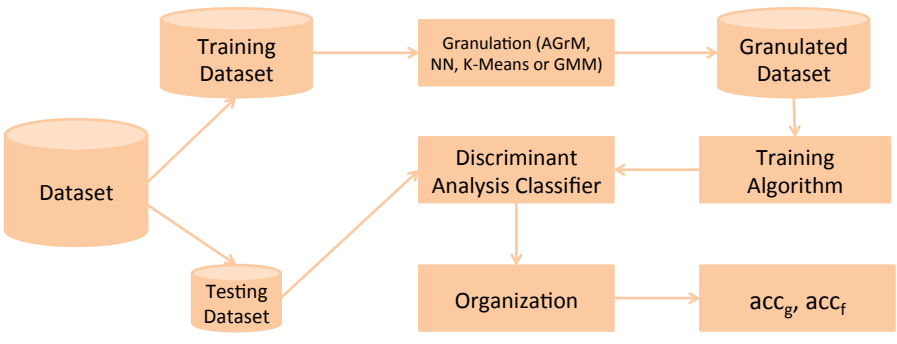

Fig. 6: Granulation-involved pattern recognition analysis.

\section{Evaluation, Results AND Discussion}

\section{A. Evaluation}

This study designed several experiments to evaluate the performance of AGrM in terms of accuracy and robustness. The first experiment was to investigate the relationship between granule number $(m)$ and the pinch recognition accuracy $\left(a c c_{g}\right)$, granule number $(m)$ and force grand recognition accuracy $\left(a c c_{f}\right)$. Meanwhile, the computing time of granulation $\left(T_{\text {granulation }}\right)$, classifier training $\left(T_{\text {traing }}\right)$ and prediction $\left(T_{\text {prediction }}\right)$ were studied with the increase of $m$. A comparison of force grand prediction accuracy on different hand pitch was conducted. The second experiences investigated the consistency of AGrM in pinch type and force grand recognition when the number of sEMG channel changes and sEMG signal were contaminated by noises. Four types of sEMG channel combination (2-channel, 4-channel, 8-channel and 16-channel), and four types of noises (10db and $-10 \mathrm{db}$ white Gaussian noise, and $10 \mu \mathrm{V}$ and $100 \mu \mathrm{V}$ power line noises). The third experiment compared AGrM with NN [26], K-Means and GMM on dataset granulation, and their impacts on pinch recognition accuracy. The time complexity of each granulation methods were also studied. The NN algorithm was implemented as described in [26]. The K-Means clustering method applied the squared Euclidean distance and selected $k$ points uniformly at random for initialisation by the use of kmeans function in Matlab 2016b. Expectation Maximization (EM) algorithm was employed to adjust the parameters of a GMM by means of likelihood maximization, and then all samples were clustered according to the probability in the GMM. The functions of fitgmdist and cluster in Matlab 2016b were combined to implement the GMM-based clustering algorithm. For K-Means and GMM, the maximum iteration times was set to 100 .

Fig. 6 demonstrated the procedure of granulation-involved pattern recognition analysis. The procedure can be described as follows. 1) divide the dataset into training and test datasets according to the standard 10-fold-cross-validation method. 2) granulate the training dataset according to the experimental requirement by means of AGrM, NN, K-Means or GMM to form a granulated dataset. 3) train a discriminant analysis classifier (the function fitcdiscr in Matlab 2016b). 4) classify the samples in testing dataset and then calculate the accuracies $\left(a c c_{g}\right.$ and $\left.a c c_{f}\right)$ by an organisation algorithm. 


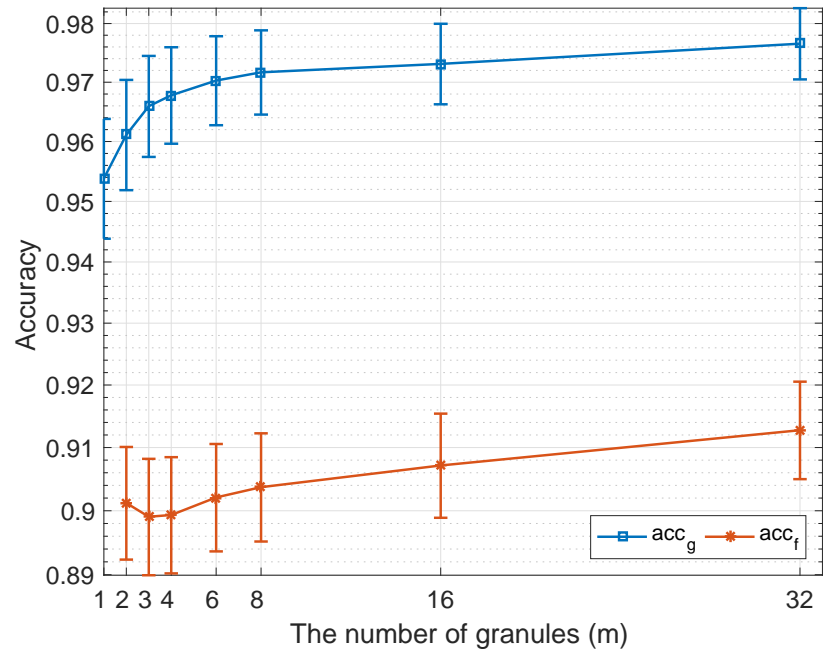

Fig. 7: The change of accuracy alone the number of granules

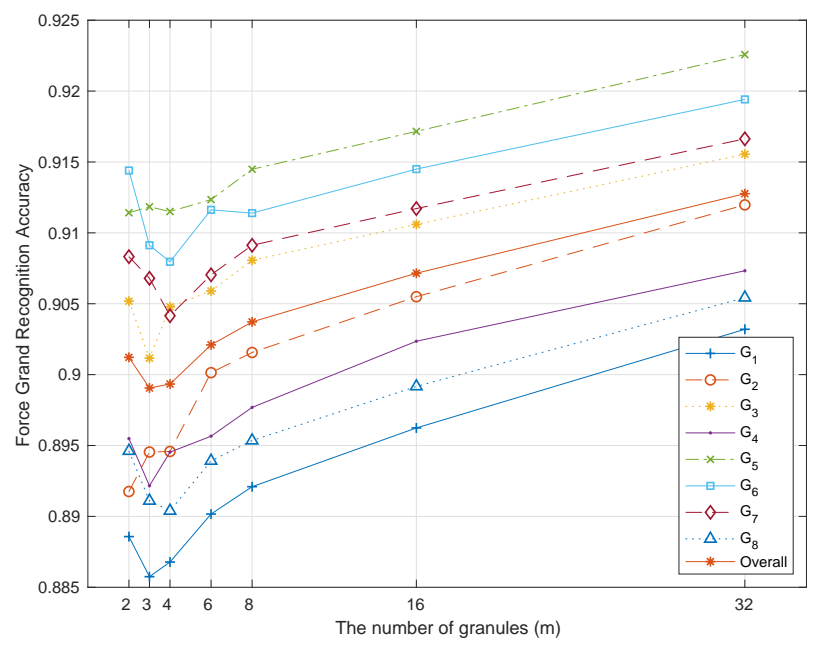

Fig. 8: A comparison of the force recognition accuracy of different pinch types.

\section{B. Accuracy}

Fig. 7 demonstrated the accuracies of $a c c_{g}, a c c_{f}$ at different numbers of granules. The error bar reflected the 0.3 times standard deviation across 10 subjects. It could be found that the $a c c_{g}$ increased significantly when $m$ was less than 8 , and tended to be stable afterwords. $a c c_{f}$ decreased when $m<=4$, followed by a steady increase.

The result indicated the effectiveness of AGrM in two perspectives. On the one hand, the pinch-type recognition accuracy $a c c_{g}$ can be improved. In the comparison between $m=1$ (i.e. without $\mathrm{AGrM}$ ) and $m=8$ (i.e. construct 8 granules for each class), it was found that the pinch-type recognition accuracy $a c c_{g}$ improved from $95.38 \pm 3.0 \%$ to $97.17 \pm 2.1 \%$ by $1.79 \%$, and this finding was statistically significant $p<0.005$ (paired t-test). Moreover, Fig. 7 also demonstrated that further accuracy rise can be obtained via adopting larger number of granules, although the improvement was very limited. It can be found that the increasing rate was $1.79 \%$ from $m=1$ to $m=8$, while it was only $0.49 \%$

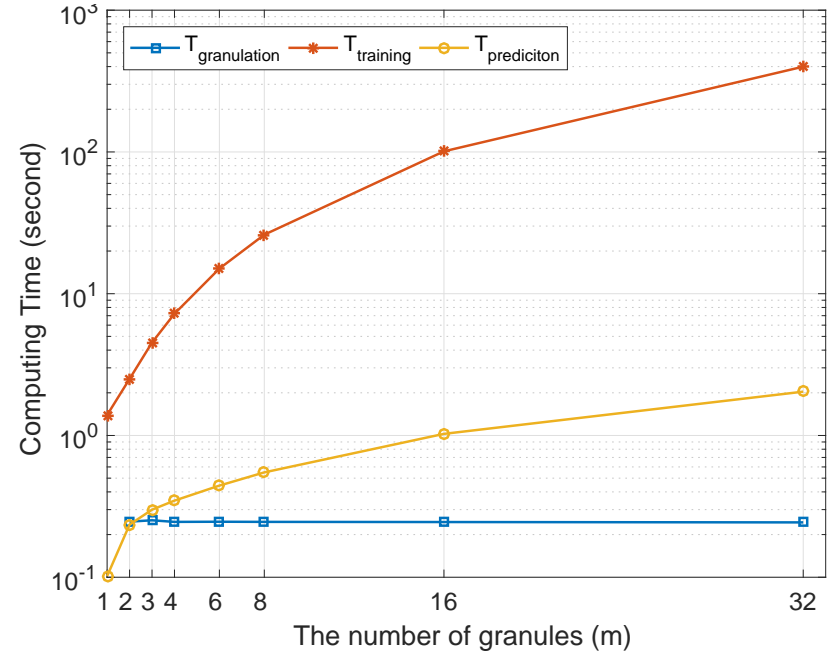

Fig. 9: The change of computing time alone the number of granules

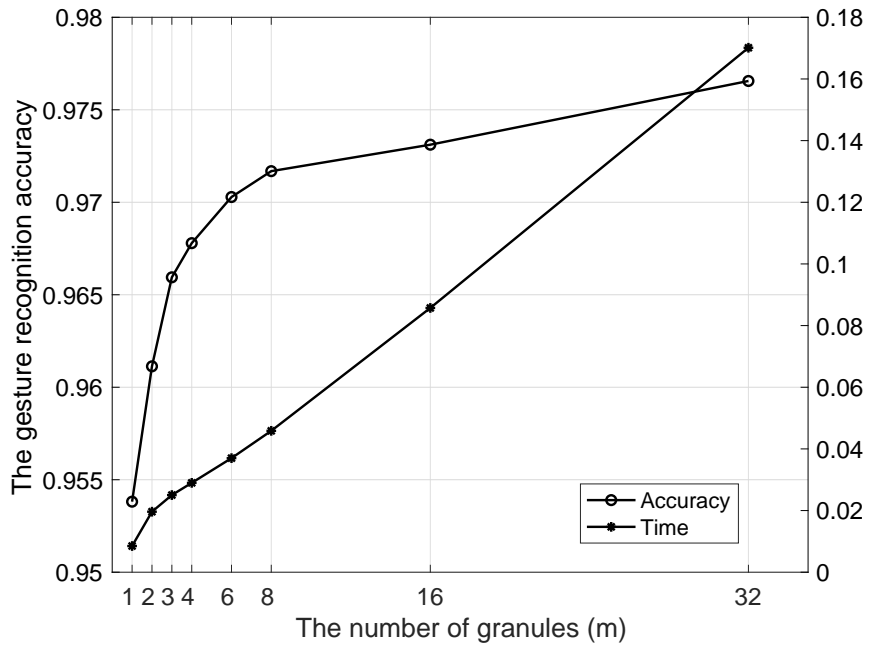

Fig. 10: A comparison demonstration on the prediction time cost and the accuracy improvement.

from $m=8$ to $m=32$. On the other hand, the fingertip force grand prediction accuracy $\left(a c c_{f}\right)$ decreased a little and then started to increase after $m=3$. The final accuracy was about $91.27 \%$ at $m=32$ in our case. Moreover, it was clear that the force estimation accuracy $\left(a c c_{f}\right)$ dropped by $0.22 \%$ in the comparison between $m=2$ and $m=3$. In despite of the accuracy decreasing, the increase of granule number improved the resolution of force grand. Fig. 8 demonstrated the force grand prediction accuracy for different pinch. It can be found that the overall accuracy change was consistent with that of each pinch type, especially when $m \geqslant 6$. The initial decreasing is possibly because that the system was of very high accuracy in predicting only two force grands, regardless the misclassification of grasping-type. This study suggests to construct at least 8 granules to form 8 grasp force grands. It can enhance grasp recognition accuracy while keep an acceptable force grands resolution and accuracy. It is worth noting that the performance can be further improved with the 


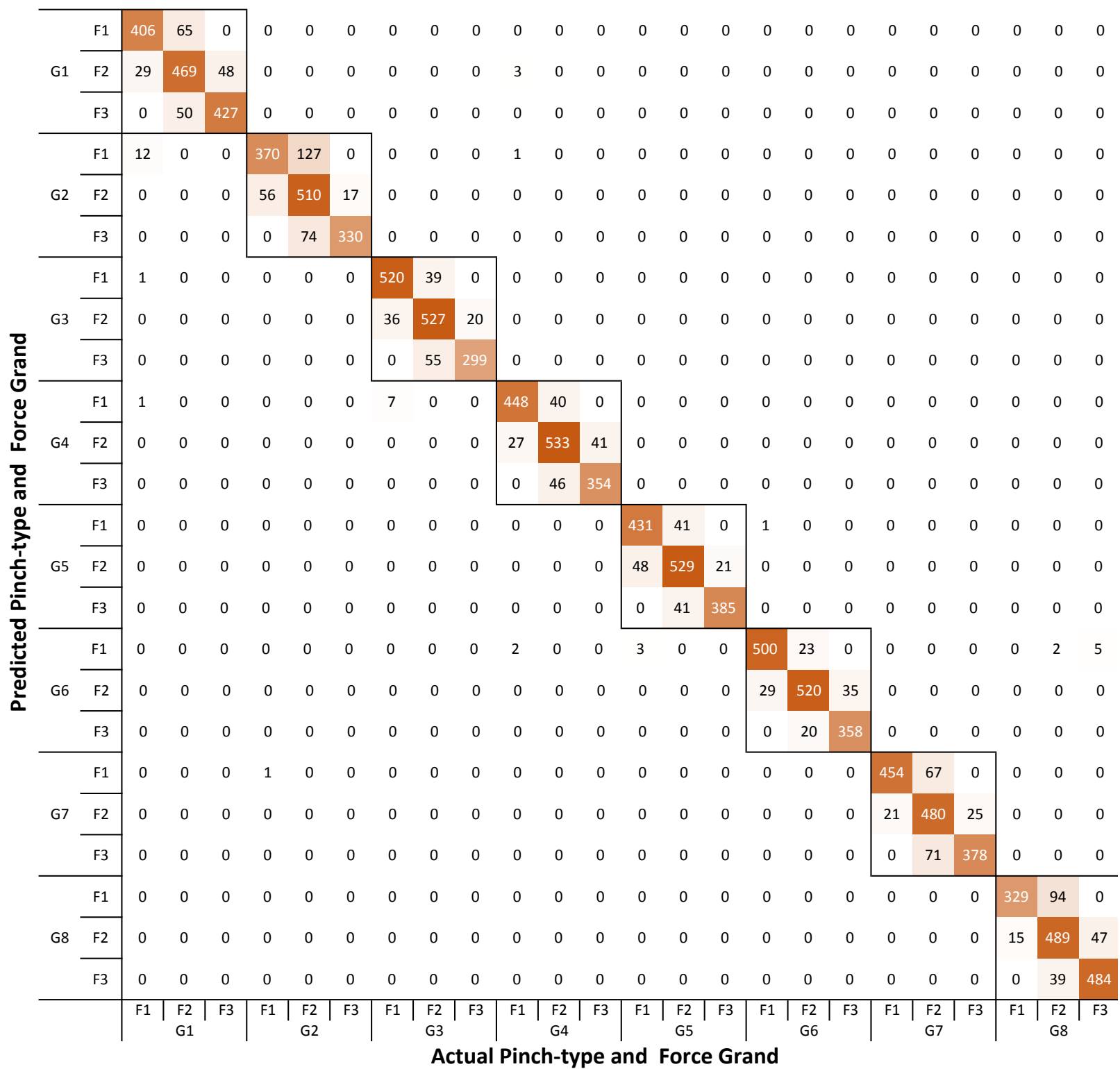

Fig. 11: The confusion matrix in the prediction of pinch-types and the fingertip force grand (from the 1st subject, $m=3$ ). G1 to G8 indicate the eight pinch-types, and F1 to F3 indicate three force grands for each pinch.

increase of $m$ as the tendency indicated in Fig. 7.

Fig. 11 demonstrated the confusion matrix for the prediction of the pinch-type and fingertip force grand, where three granules were constructed (i.e. $m=3$ ). In this example, the pinch recognition accuracy was $a c c_{g}=99.17 \%$, and the force grand prediction accuracy was $a c c_{f}=96.39 \%$. Although misclassification of both pinch-type and force grand occasionally happened, most misclassified force grand were from the same pinch. In addition, a force grand misclassified sample was very likely to be in the adjacent force grand of the correct pinch, which would avoid the larger failure cost in real prosthetic hand manipulation.

\section{Time Complexity}

The computational cost for granulation $\left(T_{\text {granulation }}\right)$, classifier training $\left(T_{\text {training }}\right)$ and prediction $\left(T_{\text {prediction }}\right)$ can be found in Fig. 9. This result was obtained through a personal computer with Windows 7 64bits system, $2.2 \mathrm{GHz}$ i5-5200U CPU and 8GB Memory, and the code was run in Matlab R2015a. The dataset was of 11976 samples and 66 dimensions (64 EMG features, 1 force attributes and 1 label ). 10-foldcross-validation was applied to accumulate the time cost. The experimental result revealed that granulation approach was time-efficient comparing with classifier training. The time complexity of classifier training and prediction was at $O\left(\mathrm{~m}^{2}\right)$ and $O(\mathrm{~km})$, respectively. As demonstrated in Fig. 10, the hand gesture classification accuracy received a logarithmic growth along the use of more number of granules, while the prediction time cost was linearly related to the number of granules, fitting the function of $y=0.06 x+0.085$, where $x$ is the number of granules and $y$ is the prediction time cost. It could be predicted that the prediction time was around 


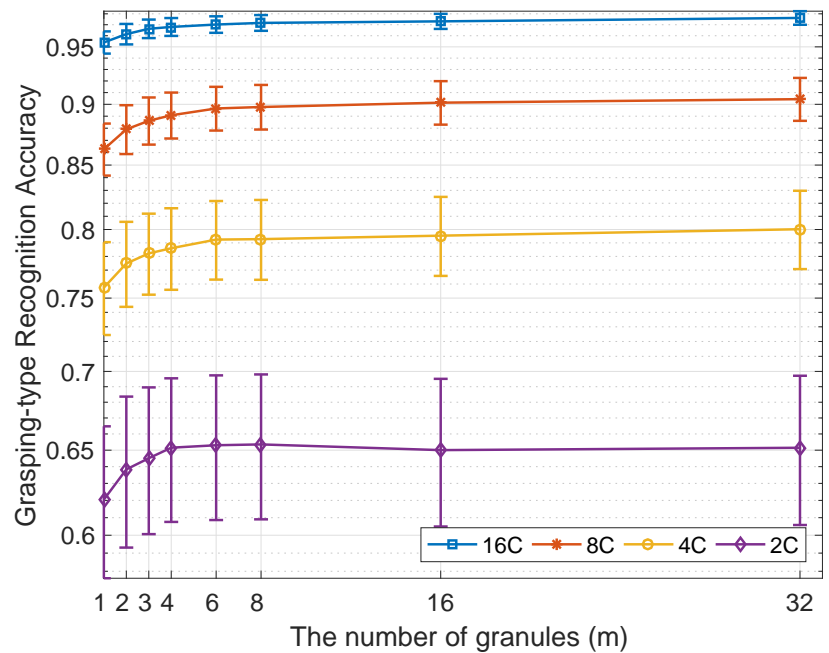

Fig. 12: The comparison of pinch-type recognition accuracy in different EMG channel combination.

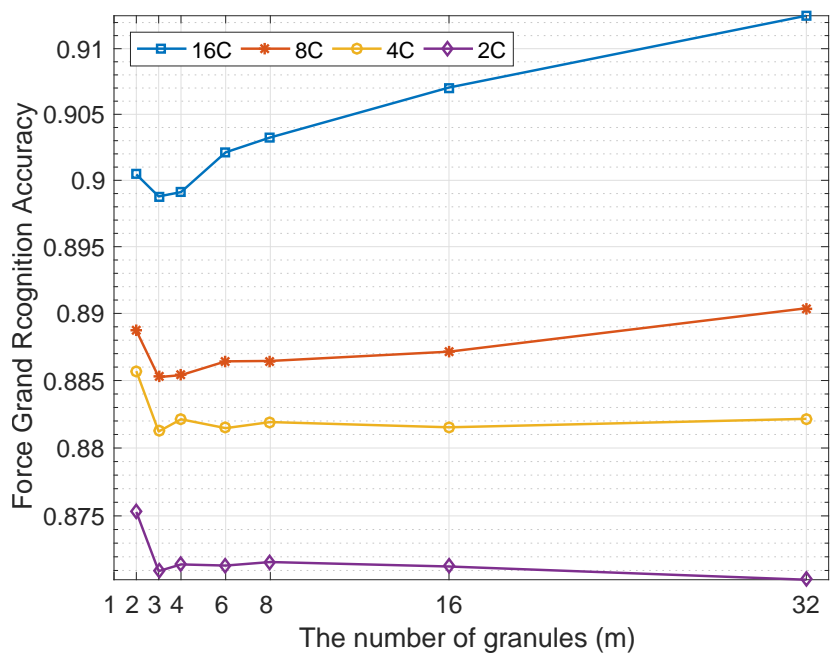

Fig. 13: The comparison of force grand estimation accuracy in different EMG channel combinations

$6 \mathrm{~ms}$ for every 100 granules, which was much lower than the $40 \mathrm{~ms}$ increments of the segmentation sliding window, satisfying the real-time performance for pinch recognition and force estimation. Thus, it is believed that the proposed granular model will not influence the efficiency of a pattern recognition based myoelectric system.

\section{Sensitivity Analysis}

Sensitivity analysis was conducted to evaluate the robustness of AGrM on EMG based pinch-type and fingertip force grand prediction. The involved factors included different channel combinations, White Gaussian noise (WGN) and $50 \mathrm{~Hz}$ Power Line Noise (PLN).

Fig. 12 compared the pinch recognition accuracy under 2, 4, 8 and 16 selected EMG channels, and it can be found that the increasing trends along $m$ were similar, indicating the adaptation of AGrM towards EMG channel number. Four curves demonstrated obvious accuracy improvement when

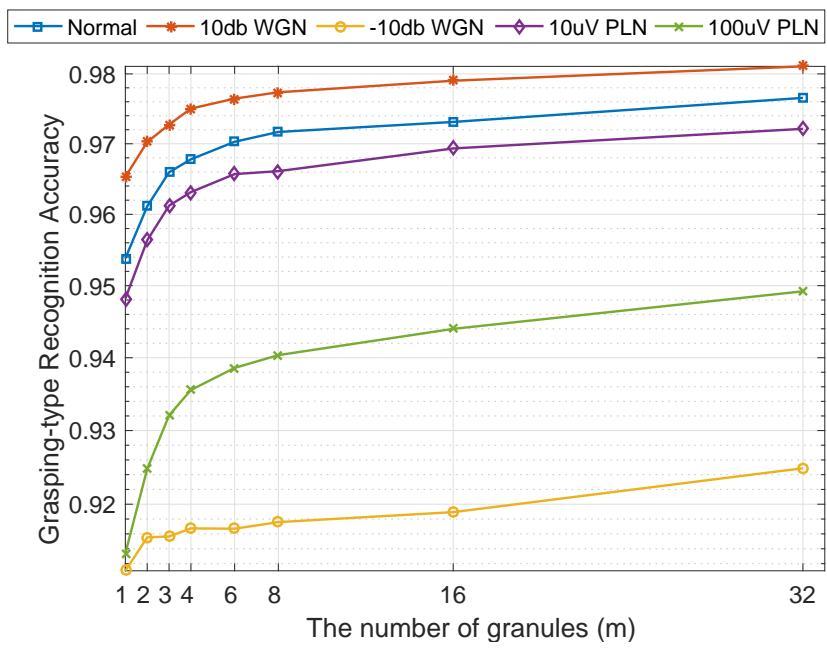

Fig. 14: The comparison of pinch-type recognition accuracy in different noise levels.

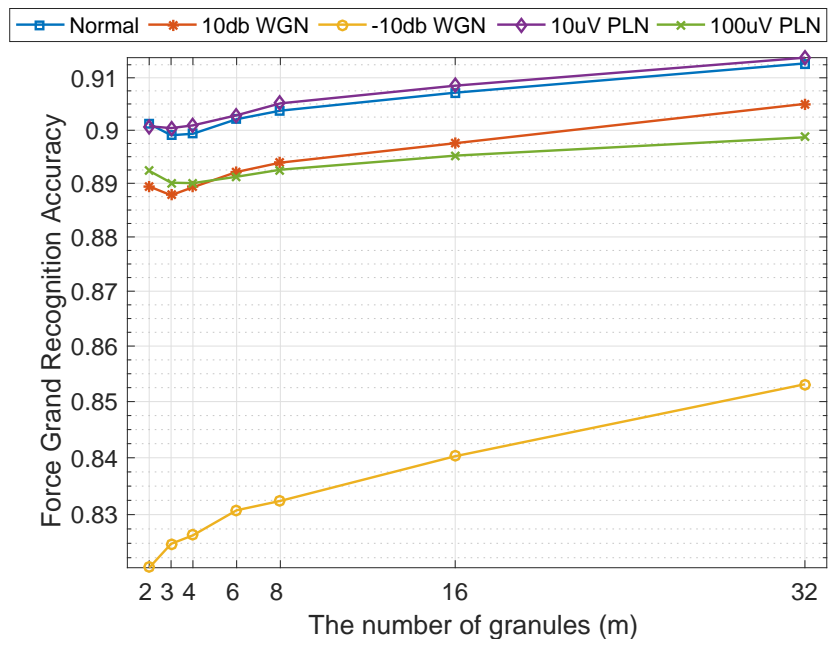

Fig. 15: The comparison of force grand estimation accuracy in different noise levels.

$m<=8$, then became stable. In addition, it was also found that the use of 16 EMG channels was somewhat necessary, which improved accuracy from $89.8 \%$ to $97.2 \%$ comparing with 8 channels. This results imply that the use of HD-sEMG configuration is promising for hand motion recognition.

Fig. 13 demonstrated the force grand prediction accuracy. Four curves indicating different channel combinations illustrated a similar tendency, which revealed that AGrM was robust towards different channel combination in EMG-based force grand recognition. These four curves demonstrated big accuracy decrease in the comparison between $m=2$ and $m=4$, and then tended to be stable. All the accuracies in Fig. 13 were above $85 \%$, indicating that the reduction of EMG channel number did not influence EMG based fingertip force grand recognition significantly.

Fig. 14 demonstrated the pinch recognition accuracies under five types of noise condition: raw EMG, white Gaussian noise contaminated sEMG at $10 \mathrm{db}$ and $-10 \mathrm{db}$ signal-to-noise ratio and $10 \mu \mathrm{V}$ and $100 \mu \mathrm{v}$ PLN contaminated EMG. Similar 


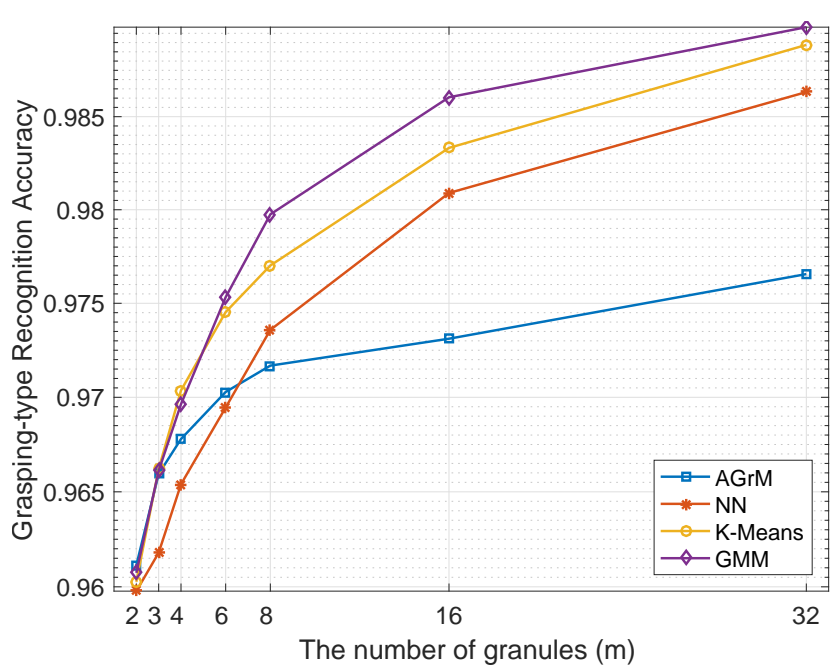

Fig. 16: The comparison of grasping-type recognition accuracy with different granulation methods.

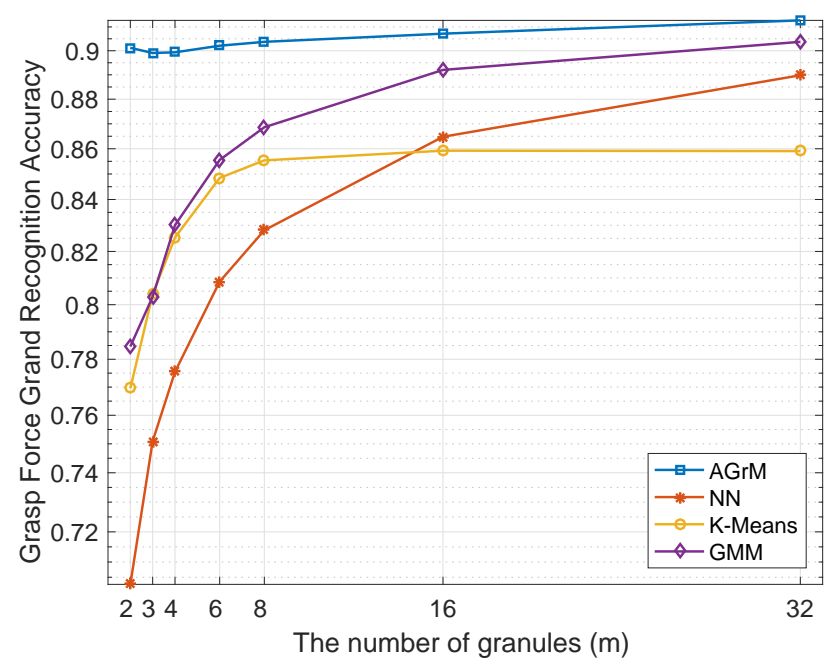

Fig. 17: The comparison of grasp force recognition accuracy with different granulation methods.

tendencies from five situations could be identified in Fig. 14, indicating the robustness of AGrM. The accuracy improvement was obvious in the comparison between $m=1$ and $m=8$. It is worth noting that $10 \mathrm{db}$ active WGN (10db WGN) increased the pinch recognition accuracy at all tested granule numbers, which was possibly because that a classifier trained by a dataset containing certain WGN noise would be more robust towards environment interference.

The comparison of force grand prediction accuracy was given in Fig. 15. The tendency of accuracy kept in good consistence, indicating the robustness of AGrM in force grand estimation towards a certain interferences. It could be also found that the inclusion of $10 \mu \mathrm{V}$ PLN contributed to the force grand prediction when $m \geqslant 4$.

\section{E. Attribute-Driven Granular Model vs Other Clustering- based Granulation Methods}

AGrM is a supervised granulation method, which granulises each class within the training dataset into several granules

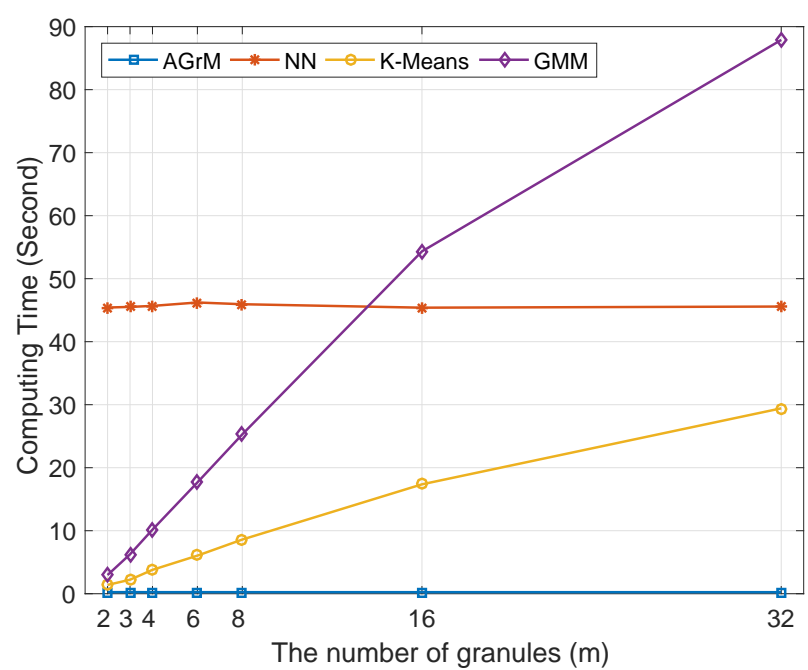

Fig. 18: The comparison of computing time for different granulation methods.

based on the simultaneously recorded sub-attributes. The subattributes was fingertip force signal in this study. Certainly, unsupervised clustering methods can also be utilized to implement the granulation task. However, unsupervised method is not able to interpret the physical meaning of each granule. AGrM is capable of solving the problem. For example, if a granule is generated under a certain force grand, then it can be said that the granule is of the property of that force grand. This study compared AGrM with NN, K-Means and GMM, and it was found that all these granulation methods improved the accuracy along with the increasing of $m$. Fig. 16 compared four types of granulation methods in terms of pinch prediction accuracy. It was found that AGrM achieved the highest accuracy $(96.11 \%)$ when $m=2$ and NN outperformed AGrM when $m \geqslant 8$. With the increase of $m(6 \leqslant m \leqslant 32)$, GMM outperformed the rest methods, and when $m$ reached to 32, GMM achieved the highest accuracy, followed by KMeans, NN, and AGrM. Figs. 19 and 20 demonstrated the granulation results on the training dataset from the first subject and pinch G1 at $m=3$ and $m=6$, respectively. This result revealed that AGrM did not show the advantage of pinch recognition accuracy against the others especially with large number of granules. To make force grand recognition accuracy comparable among these granulation methods, this study manually assigned a force grand label according to the average force value of all samples in each granule after granulation. Fig. 17 demonstrated the comparison result for force grand estimation. It was found that AGrM clearly outperformed the others.

Fig. 18 demonstrated the computing time on training dataset granulation. The time complexity for both AGrM and NN were $O(1)$, but NN costed 200 times more than AGrM. In NN algorithm, most time consumption was inevitably occupied in constructing the distance matrix. The time complexity for KMeans was $O(k m)(k \approx 0.10)$ and GMM $(k \approx 0.34)$. Overall, the AGrM presented superb time efficiency on granulation than the others. Let alone the dominant advantage of AGrM 

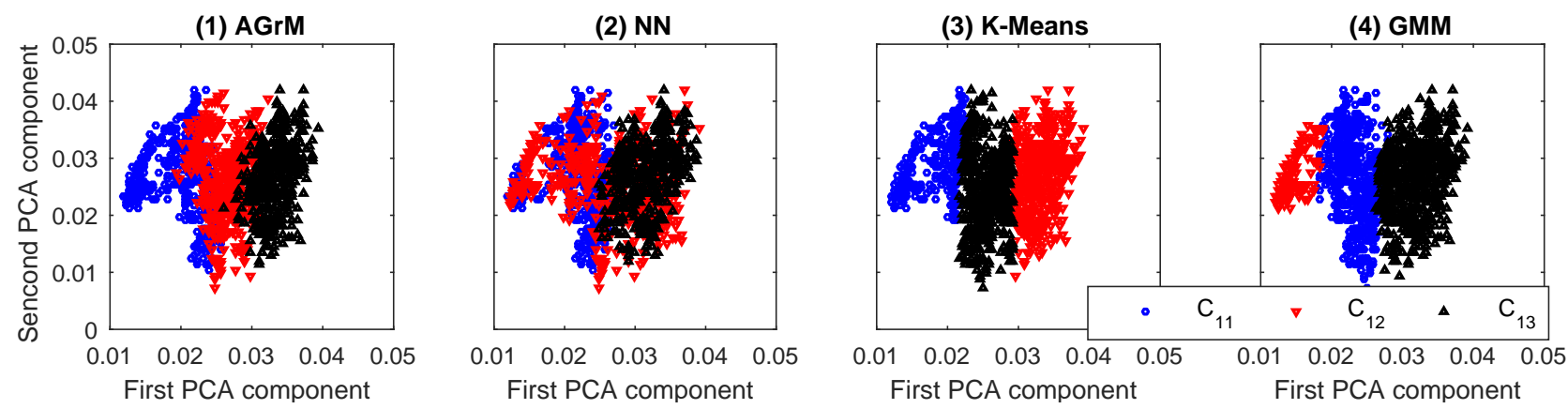

Fig. 19: The comparison of granulation effect on different granulation methods at $m=3$.
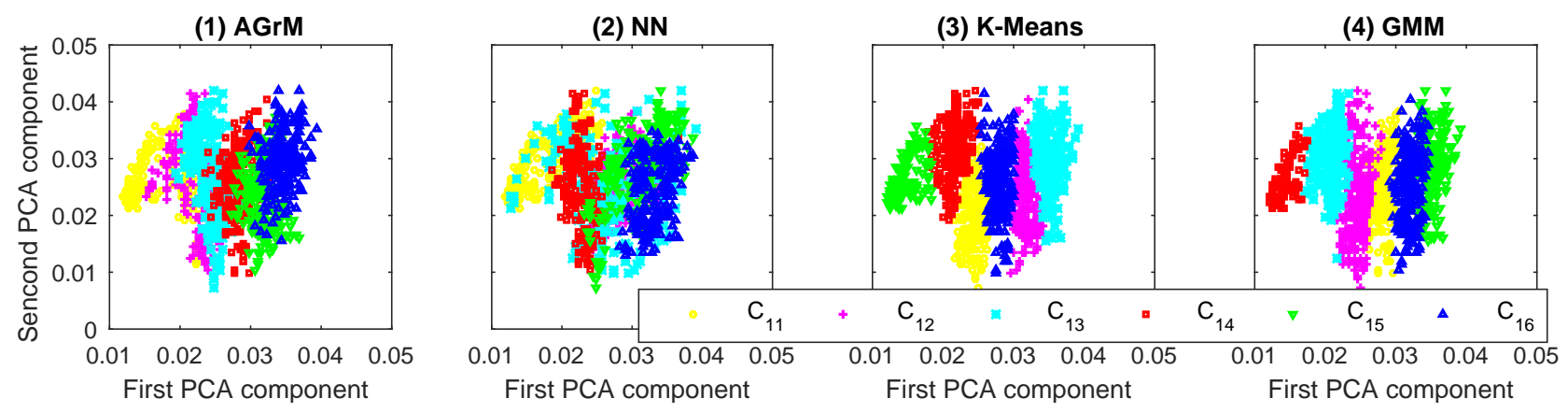

Fig. 20: The comparison of granulation performance on different granulation methods at $m=6$.

in force grand prediction, it was also benefited from the computational efficiency while preserving comparable pinch recognition accuracy to NN, K-Means and GMM.

\section{CONCLUSION AND FUTURE WORK}

This paper proposed an AGrM to enhance EMG based hand pinch and force grand recognition, which can be applied to fine prosthetic hand manipulation in a unified machine learning framework. We introduced the background of granular computing in machine learning, and then theoretically presented the AGrM in the case of hand motion recognition with the EMG signal as the main-attribute and the force as the sub-attribute. EMG signals and fingertip force signals were recorded synchronously from 8 subjects to evaluate the effectiveness of AGrM in terms of pinch-type recognition accuracy, and fingertip force grand prediction accuracy in different conditions. Our results demonstrated its advantages on the following aspects: 1) red pinch-type recognition accuracy logarithmically grew with the rising number of granules $m$; 2) force grand prediction accuracy increased with the rising number of granules when $m \geqslant 3$; 3) AGrM was insensitive to different EMG channel combinations, and noises; 4) AGrM was of the lowest computational cost on training dataset granulation in the comparison with NN, K-Means and GMM, and could provide comparable pinch-type recognition accuracy and higher force grand recognition accuracy. In the theoretical view of information granulation, AGrM provided supplement to traditional unsupervised granulation method, where semantic explanation for each granule was missing. In the future, AGrM will be tested in an on-line environment to predict more hand motion as well as its force grand for prosthetic hand manipulation via decoding EMG signal. Besides, AGrM will be also tested in a wider range of machine learning scenarios.

\section{REFERENCES}

[1] K. Kiguchi, S. Kariya, K. Watanabe, K. Izumi, and T. Fukuda, "An exoskeletal robot for human elbow motion support-sensor fusion, adaptation, and control," IEEE Trans. Syst., Man, Cybern. B, Cybern., vol. 31, no. 3, pp. 353-361, 2001.

[2] K. Kiguchi and Y. Hayashi, "An emg-based control for an upper-limb power-assist exoskeleton robot," IEEE Trans. Syst., Man, Cybern. B, Cybern., vol. 42, no. 4, pp. 1064-1071, 2012.

[3] E. Scheme and K. Englehart, "Electromyogram pattern recognition for control of powered upper-limb prostheses: State of the art and challenges for clinical use," J. Rehabil. Res. Dev., vol. 48, no. 6, pp. 643-659, 2011.

[4] B. Peerdeman, D. Boere, H. Witteveen, H. Hermens, S. Stramigioli, J. Rietman, P. Veltink, S. Misra et al., "Myoelectric forearm prostheses: State of the art from a user-centered perspective," 2011.

[5] H. Liu, "Exploring human hand capabilities into embedded multifingered object manipulation," IEEE Trans. Ind. Informat., vol. 7, no. 3, pp. 389398, 2011.

[6] Z. Ju and H. Liu, "Human hand motion analysis with multisensory information," IEEE/ASME Trans. Mechatronics, vol. 19, no. 2, pp. 456466, 2014

[7] J. Y. He, D. G. Zhang, X. J. Sheng, S. C. Li, and X. Y. Zhu, "Invariant surface emg feature against varying contraction level for myoelectric control based on muscle coordination," IEEE J. Biomed. Health Inform., vol. 19, no. 3, pp. 874-882, 2015.

[8] M. Powell, R. R. Kaliki, N. V. Thakor et al., "User training for pattern recognition-based myoelectric prostheses: Improving phantom limb movement consistency and distinguishability," IEEE Trans. Neural Syst. Rehabil. Eng., vol. 22, no. 3, pp. 522-532, 2014.

[9] D. Tkach, H. Huang, and T. A. Kuiken, "Study of stability of timedomain features for electromyographic pattern recognition," J. Neuroeng. Rehabil., vol. 7, no. 1, p. 1, 2010.

[10] N. M. Kakoty, S. M. Hazarika, and J. Q. Gan, "Emg feature set selection through linear relationship for grasp recognition," Journal of Medical and Biological Engineering, vol. 36, no. 6, pp. 883-890, 2016.

[11] Y. Fang, D. Zhou, K. Li, Z. Ju, and H. Liu, "A force-driven granular model for emg based grasp recognition," in 2017 IEEE International Conference on Systems, Man, and Cybernetics (SMC), Oct 2017, pp. 2939-2944. 
[12] Y. Fang, D. Zhou, K. Li, and H. Liu, "Interface prostheses with classifierfeedback based user training," IEEE Trans. Biomed. Eng., vol. PP, no. 99, pp. 1-9, 2016.

[13] C. Wu, H. Zeng, A. Song, and B. Xu, "Grip force and 3d push-pull force estimation based on semg and grnn," Frontiers in neuroscience, vol. 11, p. 343, 2017.

[14] D. Yang, J. Zhao, Y. Gu, L. Jiang, and H. Liu, "Estimation of hand grasp force based on forearm surface emg," in Proc. Int. Conf. Mechatron. Autom., 2009, pp. 1795-1799.

[15] C. Castellini and P. van der Smagt, "Surface emg in advanced hand prosthetics," Biol. Cybern., vol. 100, no. 1, pp. 35-47, 2009.

[16] D. Yang, J. Zhao, Y. Gu, L. Jiang, and H. Liu, "Emg pattern recognition and grasping force estimation: Improvement to the myocontrol of multidof prosthetic hands," in Proc. Int. Conf. Intell. Robot. Syst., 2009, pp. 516-521.

[17] J. T. Yao, A. V. Vasilakos, and W. Pedrycz, "Granular computing: perspectives and challenges," IEEE Trans. Cybern., vol. 43, no. 6, pp. 1977-1989, 2013.

[18] V. J. Kok and C. S. Chan, "Grcs: Granular computing-based crowd segmentation," IEEE Trans. Cybern., pp. 1-12, 2016.

[19] T. Wang, Z. Han, J. Zhao, and W. Wang, "Adaptive granulation-based prediction for energy system of steel industry," IEEE Trans. Cybern., pp. 1-12, 2016.

[20] J. Zhao, Z. Han, W. Pedrycz, and W. Wang, "Granular model of long-term prediction for energy system in steel industry," IEEE Trans. Cybern., vol. 46, no. 2, pp. 388-400, 2016.

[21] S. K. Pal and D. B. Chakraborty, "Granular flow graph, adaptive rule generation and tracking," IEEE Trans. Cybern., pp. 1-12, 2016.

[22] H. Zhang, W. Pedrycz, D. Miao, and Z. Wei, "From principal curves to granular principal curves," IEEE Trans. Cybern., vol. 44, no. 6, pp. 748-760, 2014

[23] H. Liu, C. Liu, and C. A. Wu, "Granular computing classification algorithms based on distance measures between granules from the view of set," Comput. Intell. Neurosci., vol. 2014, p. 656790, 2014.

[24] S.-B. Roh, W. Pedrycz, and T.-C. Ahn, "A design of granular fuzzy classifier,” Expert. Syst. Appl., vol. 41, no. 15, pp. 6786-6795, 2014.

[25] Y. Tang, B. Jin, Y. Sun, and Y.-Q. Zhang, "Granular support vector machines for medical binary classification problems," in Proc. Symp. Compu. Intell. BioInform. Compu. Biology. IEEE, 2004, pp. 73-78.

[26] M. L. Zhu and A. M. Martinez, "Subclass discriminant analysis," IEEE Trans. Pattern Anal. Mach. Intell., vol. 28, no. 8, pp. 1274-1286, 2006.

[27] A. Bargiela and W. Pedrycz, "Granular mappings," IEEE Trans. Syst., Man, Cybern. A, Syst.,Humans, vol. 35, no. 2, pp. 292-297, 2005.

[28] L. A. Zadeh, "Toward a theory of fuzzy information granulation and its centrality in human reasoning and fuzzy logic," Fuzzy Set Syst., vol. 90, no. 2, pp. 111-127, 1997.

[29] S. Di Cataldo, A. Bottino, I. Ul Islam, T. F. Vieira, and E. Ficarra, "Subclass discriminant analysis of morphological and textural features for hep-2 staining pattern classification," Pattern Recogn., vol. 47, no. 7, pp. 2389-2399, 2014.

[30] S. W. Kim, "A pre-clustering technique for optimizing subclass discriminant analysis," Pattern Recogn. Lett., vol. 31, no. 6, pp. 462-468, 2010.

[31] A. Pnevmatikakis and L. Polymenakos, "Subclass linear discriminant analysis for video-based face recognition," J. Vis. Commun. Image R., vol. 20, no. 8, pp. 543-551, 2009.

[32] B. Mandal, L. Li, V. Chandrasekhar, and J. H. Lim, "Whole space subclass discriminant analysis for face recognition," in Proc. Inter. Conf. Image Process., 2015, pp. 329-333.

[33] X. Zhu, W. Pedrycz, and Z. Li, "Granular data description: Designing ellipsoidal information granules," IEEE Trans. Cybern., pp. 1-10, 2016.

[34] W. Pedrycz, R. Al-Hmouz, A. Morfeq, and A. Balamash, "The design of free structure granular mappings: the use of the principle of justifiable granularity," IEEE Trans. Cybern., vol. 43, no. 6, pp. 2105-2113, 2013.

[35] W. Pedrycz and A. Bargiela, "An optimization of allocation of information granularity in the interpretation of data structures: toward granular fuzzy clustering," IEEE Trans. Syst., Man, Cybern. B, Cybern., vol. 42, no. 3, pp. 582-590, 2012.

[36] Z. Ju and H. Liu, "A unified fuzzy framework for human-hand motion recognition,” IEEE Trans. Fuzzy Syst., vol. 19, no. 5, pp. 901-913, 2011.

[37] Y. Fang, N. Hettiarachchi, D. Zhou, and H. Liu, "Multi-modal sensing techniques for interfacing hand prostheses: A review," IEEE Sens. J, vol. 15, no. 11, pp. 6065-6076, 2015.

[38] Y. Yao, "Granular computing: basic issues and possible solutions," in Proc. 5th Joint Conf. Inf. Sci., 2000, pp. 186-189.

[39] J. V. Basmajian and C. De Luca, "Muscles alive," Muscles alive: their functions revealed by electromyography, vol. 278, p. 126, 1985.
[40] T. Feix, J. Romero, H.-B. Schmiedmayer, A. M. Dollar, and D. Kragic, "The grasp taxonomy of human grasp types," IEEE Transactions on human-machine systems, vol. 46, no. 1, pp. 66-77, 2015.

[41] B. Hudgins, P. Parker, and R. N. Scott, "A New Strategy for Multifunction Myoelectric Control," IEEE Transactions on Biomedical Engineering, vol. 40, no. 1, pp. 82-94, 1993.

[42] Y. Fang, "Interacting with prosthetic hands via electromyography signals," Ph.D. dissertation, University of Portsmouth, 2015.

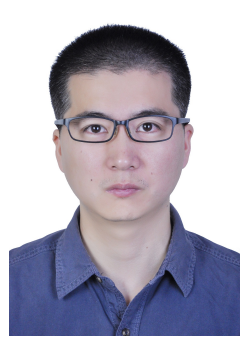

Yinfeng Fang (S'12-M'15) received the Ph.D. degree in Computer Science from the University of Portsmouth, Portsmouth, U.K., in 2015. He presently is an Associate Professor in the School of Communication Engineering, Hangzhou Dianzi University, Hangzhou, China. He previously held a research appointment at the University of Portsmouth.

Dr. Fang has published more than 30 peer-reviewed international journal and conference papers. He is also Guest Editor of the International Journal of Humanoid Robotics. His research interests include bioelectric signals acquisition, signal processing and artificial intelligence algorithms, and neuromuscular rehabilitation.

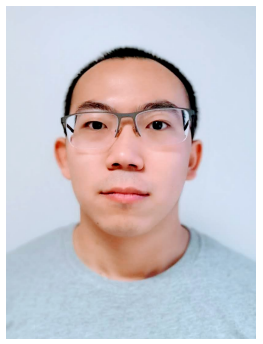

Dalin Zhou (S'15) received the B.S. degree in Automation from University of Science and Technology of China in 2012, and the Ph.D. degree in Computing from the University of Portsmouth, Portsmouth, U.K. in 2018. He currently is a lecturer in Computer Science with the School of Computing at University of Portsmouth, Portsmouth, U.K. His research interests are signal processing, machine learning and multi-modal fusion.

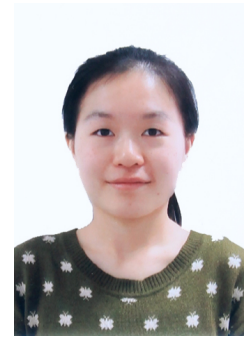

Kairu Li (S'15) received the Ph.D. degree in intelligent robotics from the University of Portsmouth, Portsmouth, U.K., in 2018. She previously received the M.S. degree in Control Theory and Control Engineering and the B.S. degree in Automation from Dalian University of Technology, China, in 2015 and 2012. She is currently an associate professor in Automation in the School of Electrical Engineering, Shenyang University of Technology. Her research interests include wearable sensor systems, electrotactile stimulation, sensory feedback in prosthetics

and rehabilitation.

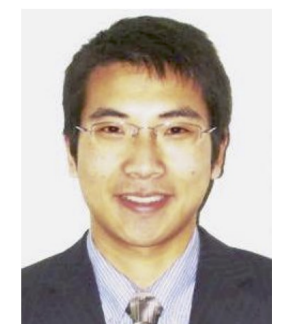

Zhaojie Ju (M'08-SM'16) received the B.S. in automatic control and the M.S. in intelligent robotics both from Huazhong University of Science and Technology, China, and the Ph.D. degree in intelligent robotics at the University of Portsmouth, UK.

He held a research appointment at the University College London, London, U.K., before he started his independent academic position at the University of Portsmouth, U.K., in 2012. His research interests include machine intelligence, pattern recognition, and their applications on human motion analysis, multi-fingered robotic hand control, human-robot interaction and collaboration, and robot skill learning. He has authored or co-authored over 170 publications in journals, book chapters, and conference proceedings and received four Best Paper Awards and one Best AE Award in ICRA2018.

$\mathrm{Dr}$. Ju is an Associate Editor of the IEEE TRANSACTIONS ON CYBERNETICS, Journal of Intelligent \& Fuzzy Systems, International Journal of Fuzzy Systems, and Chinese Journal of Mechanical Engineering. 


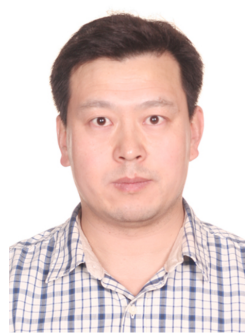

Honghai Liu (M'02-SM'06) received the Ph.D. in intelligent robotics from King's College London, London, U.K., in 2003. He is currently Chair Professor of Intelligent Systems and Robotics, University of Portsmouth, Portsmouth, U.K., and is also Professor in School of Mechanical Engineering, Shanghai Jiao Tong University, Shanghai, China. His research interests include wearable sensing, biomechatronics, intelligent video analytics, intelligent robotics, and their applications with an emphasis on approaches that could make contributions to the intelligent connection of perception to action using contextual information.

Prof. Liu is a fellow of the Institution of Engineering and Technology. He is also the Associate Editor of IEEE Transactions on Industrial Informatics, and IEEE Transactions on Industrial Electronics. 